\section{Polymer Nanotubes by Wetting of Ordered Porous Templates}

\author{
M. Steinhart, ${ }^{1,2}$ J. H. Wendorff, ${ }^{1}$ A. Greiner, ${ }^{1}$ R. B. Wehrspohn, ${ }^{2 *}$ \\ K. Nielsch, ${ }^{2}$ J. Schilling, ${ }^{2}$ J. Choi, ${ }^{2}$ U. Gösele ${ }^{2}$
}

We have developed a simple technique for the fabrication of polymer nanotubes with a monodisperse size distribution and uniform orientation. When either a polymer melt or solution is placed on a substrate with high surface energy, it will spread to form a thin film, known as a precursor film, similar to the behavior of low molar mass liquids $(1,2)$.

Similar wetting phenomena occur if porous templates are brought into contact with polymer solutions or melts: A thin surface film will cover the pore walls in the initial stages of wetting. This is because the cohesive driving forces for complete filling are much weaker than the adhesive forces. Wall wetting and complete filling of the pores thus take place on different time scales. The latter is prevented by thermal quenching in the case of melts or by solvent evaporation in the case of solutions, thus preserving a nanotube structure. If the template is of monodisperse size distribution, aligned or ordered, so are the nanotubes, and ordered polymer nanotube arrays can be obtained if the template is removed. Any melt-processible polymer, such as polytetrafluoroethylene (PTFE), blends, or multicomponent solutions can be formed into nanotubes with a wall thickness of a few tens of nanometers. Owing to its versatility, this approach should be a promising route toward functionalized polymer nanotubes.

We used ordered porous alumina and oxidized macroporous silicon templates with narrow pore size distribution (3). Extended regular pore arrays were prepared by lithography. The pores are well-defined, straight, with a smooth inner surface and with diameters $D_{P}$ between 300 and $900 \mathrm{~nm}$. To process melts, we placed the polymer on a pore array at a temperature well above its glass transition temperature, in the case of amorphous polymers, or its melting point, in the case of partially crystalline polymers. The liquid polymer forms a thin wetting film covering the entire pore surface on a time scale ranging from a few minutes to half an hour. Polymer solutions were dropped on the templates at ambient conditions (fig. S1A) (4). The resulting nanotubes obtained from either method had wall thicknesses between 20 and 50 $\mathrm{nm}$ and lengths of up to $100 \mu \mathrm{m}$. Oligomers as well as polymers with molecular masses $M_{n}$ up to several hundreds of thousands of grams per mol were processed. Figure 1 depicts nanotubes formed from several polymers by melt-wetting. The tip of a polystyrene (PS) nanotube $\left(M_{n} \sim\right.$ $850,000 \mathrm{~g} / \mathrm{mol}$ ) formed in an alumina template was uncovered by etching the alumina substrate with aqueous potassium hydroxide (Fig. 1A) (4). Figure 1B shows the same sample after the complete removal of the template. Figure 1C depicts an array of aligned PTFE nanotubes obtained by wetting an alumina template and Fig. 1D a highly

ordered array of polymethyl methacrylate (PMMA; $M_{n} \sim 80,000 \mathrm{~g} / \mathrm{mol}$ ) nanotubes prepared by wetting a macroporous silicon template. After selectively dissolving the template, the remaining nanotube array still exhibits its hexagonal long-range order.

The wetting technique can be easily extended to prepare functionalized nanotubes, for example, palladium/polymer composite nanotubes. We first wetted the porous templates with a solution containing poly-L-lactide (PLLA) and palladium(II)acetate under ambient conditions. After evaporation of the solvent dichloromethane, a PLLA/palladium(II)acetate film covered the pore walls. The template was subsequently annealed in vacuum at temperatures of up to $300^{\circ} \mathrm{C}$ to degrade PLLA (5) and to reduce $\mathrm{Pd}$. In a second wetting step, molten PS was added, so that Pd/PS composite tubes were formed (fig. S1B). Energydispersive $\mathrm{X}$-ray microanalysis verified the presence of Pd (fig. S1C), and selected area electron diffraction of single composite tubes revealed that it was metallic with a typical crystallite size of 2 to 3 nm (fig. S1D). As demonstrated by this example, template-wetting should have an outstanding potential in providing customized nanotubes for a broad range of applications in nanoscience.

References and Notes

1. P. G. de Gennes, Rev. Mod. Phys. 57, 827 (1985).

2. S. F. Kistler, in Wettability, Surfactant Science Series, vol. 49, J. C. Berg, Ed. (Dekker, New York, 1993), chap. 6.

3. R. B. Wehrspohn, J. Schilling, MRS Bull. 8, 623 (2001).

4. Materials and methods are available as supporting material on Science Online.

M. Bognitzki et al. Adv, Mater. 12, 637 (2000).

6. Support from the Deutsche Forschungsgemeinschaft (WE 2637/1-1 and WE 496/19-1) and transmission electron microscopy investigations by $\mathrm{C}$. Dietzsch are gratefully acknowledged.

\section{Supporting Online Material}

www.sciencemag.org/cgi/content/full/296/5575/1997/ DC1

Materials and Methods

Fig. S1

${ }^{1}$ Institute of Physical Chemistry and Center of Materials Science, Philipps-University, Hans-MeerweinStrasse, D-35032 Marburg, Germany. ${ }^{2}$ Max-PlanckInstitute of Microstructure Physics, Weinberg 2, D-06120 Halle, Germany.

*To whom the correspondence should be addressed. Email: wehrspoh@mpi-halle.de 